Check for updates

Hamilton, Ontario

Cite this as: BMJ 2020;370:m3340 http://dx.doi.org/10.1136/bmj.m3340 Published: 26 August 2020

\section{Covid-19: Hong Kong scientists report first confirmed case of reinfection}

Jane Parry

A 33 year old man from Hong Kong is reported to have the first confirmed case of covid reinfection.

Researchers from the University of Hong Kong's Li Ka Shing Faculty of Medicine Department of Microbiology found that the patient's two episodes were caused by virus strains with clearly different genome sequences. Their findings have not yet been published but were accepted for publication in the journal Clinical Infectious Diseases on 24 August.

"The man first acquired this infection in March, locally, probably from a colleague who travelled from London to work with him. At that time he had very mild symptoms and tested positive for covid-19," said Ivan Fan-Ngai Hung, research team member and clinical professor in the university's Department of Medicine.

"When he was hospitalised three or four days later he was already asymptomatic-all confirmed positive cases of covid-19 in Hong Kong are hospitalised for observation, symptomatic treatment, and prevention of onward transmission - and remained in hospital for three weeks until he tested negative twice," Hung added. "After that, he was very well until four and a half months later, when he came back to Hong Kong having been in Spain for a week, and he was tested on return, because everyone gets tested on arrival in Hong Kong. He was asymptomatic but still tested positive and had quite a high viral load.”

\section{"Short lived" immunity}

A press release from the team said that a total of 24 nucleotides differed between the viruses from the first and second episode. Amino acid differences were found in nine proteins, including a 58 amino acid truncation of ORF8 protein that was present only in the virus from the first infection. The findings suggest that acquired immunity after natural infection may be short lived.

"Vaccination should still be considered for those with previous infection," said Hung. He noted that thee vaccines under development were "pretty safe from this kind of mutation. But you never know: the virus could change significantly-so much so that those vaccines currently under trial may not work.”

He said that the evidence of reinfection should not be surprising, a view echoed by other observers.

Paul Hunter, professor in medicine at the University of East Anglia, UK, said, "It should not be too surprising. It is, however, important that this is documented. Commentators have been saying for some time that immunity is unlikely to be permanent and may only last a few months.

"Given the different intensity of the antibody response in people with mild or severe illness and the subsequent decay in levels, it is likely that those with a mild illness will have a shorter duration of immunity than those with severe illness."

Regarding the implications for vaccine research, Brendan Wren, professor of microbial pathogenesis at the London School of Hygiene and Tropical Medicine, said, "With over three million cases of covid-19 worldwide, the first reported case of a potential reinfection with SARS-CoV-2 needs to be taken into context. It is to be expected that the virus will naturally mutate over time. This is a very rare example of reinfection, and it should not negate the global drive to develop covid-19 vaccines." 